\title{
Role of Third Body on Bolted Joints' Self-Loosening
}

\author{
Komla Apélété Kounoudji ${ }^{1} \cdot$ Mathieu Renouf ${ }^{2} \cdot$ Guilhem Mollon $^{1} \cdot$ \\ Yves Berthier ${ }^{1}$
}

\begin{abstract}
Bolted joints are frequently subjected to selfloosening (gradual loss of clamping force) causing multiple failures, especially leaking and breaking of mechanical systems. Such physical phenomena would occur whatever the considered coating ( $\mathrm{Ag}, \mathrm{MoS}_{2}, \mathrm{Zn}-\mathrm{Ni}$ and others). To enlighten this phenomenon, which remains rather misunderstood due to the confined nature of bolted joint contacts, a coupled experimental-numerical approach is adopted on a bolted joint with silver coating. Indeed, from tribological expert assessments of disassembled joints without loosening, a local view of nut/screw threads contacts is proposed, using discrete element method. This method becomes essential in tribology since it offers the ability to model the dynamic behavior of a contact interface. The model is based on a Non-Smooth Contacts Dynamics approach. The case of third body formed in contacts during tightening process, which has been ignored so far, is placed at the focus of self-loosening phenomenon.
\end{abstract}

Keywords Third body - Silver coating - SEM analysis · DEM · Bolted joints · Self-loosening

Komla Apélété Kounoudji

komla-apelete.kounoudji@insa-lyon.fr

1 LaMCoS, INSA Lyon, UMR 5259, Université de Lyon, CNRS, 69621 Villeurbanne Cedex, France

2 Laboratoire de Mécanique et Génie Civil (LMGC), Université de Montpellier, CNRS, Montpellier, France

\section{Introduction}

The bolted joint is the most common element to efficiently assemble two plates while providing a possibility of disassembling. This is probably the reason why it represents $70 \%$ of the worldwide mechanical links [1]. Such an element is always related to a given coating which can be composed of $\mathrm{MoS}_{2}$ [2, 3], $\mathrm{Zn}-\mathrm{Ni}[4,5]$, etc. The use of another type of coating, especially Ag coating, comes now to replace $\mathrm{Cd}$ coating formerly used for fasteners operating in high-temperature applications and prohibited today by the new REACH (Registration, Evaluation, Authorization and Restriction of Chemicals) directives.

Whatever their coating, shape or size, fasteners are still used in the same goals: achieve sustainable assemblies and ensure their structural integrity. For the previous reasons, it is crucial that the clamping force be controlled and maintained over time. However, bolted joints, whether in aeronautics, aerospace, automotive or in railways engineering, are frequently subjected to gradual loss of the clamping force due to dynamic loadings. This loss is noticed in terms of self-loosening of assemblies [6, 7] causing their cracking or even breaking [8].

To better understand self-loosening of bolted joints and provide solutions, many works have been conducted, based on experimental or numerical aspects. However, the phenomenon remains until today unexplained, because it can occur with or without rotation of the nut.

Experimentally, Junker testings [9] are usually performed to detect categories of bolted joints likely to resist any longer to self-loosening phenomenon. In this case, the phenomenon occurs by a gradual rotation of the nut. In complement, torque-tension testings enable to measure preload and friction values related to different interfaces of the assemblies based on empirical [10-13] and analytical 
[14] formulas. Indeed, Eccles [7] made torque-tension testings at several cycles of tightening/loosening process with the same specific torque value and found that the measured preload decreases according to the number of cycles. By achieving tribological observations after the last cycle, he concludes that the degradation of the threads coating is the cause of the gradual loss of the clamping force under dynamic loadings.

From a numerical point of view, bolted joints are modeled at a macroscopic scale, using finite element method approaches essentially. In such models, the contacts are supposed perfect $[15,16]$, and all aspects related to friction, in threads for example, are reduced to a famous friction parameter $\mu$. Although this parameter is essential because it can encompass all tribological phenomena that take place in contact ensuring shear strength, it completely hides the complexity and the understanding of the underlying problem. Following a different approach, Jiang and al. [16], after an overview of the problem, have conducted elastic-plastic finite element analysis and come to the conclusion that, under dynamic loadings, local cyclic plasticity occurring near the roots of the engaged threads is the initiator of the gradual loss of the clamping force. However, in a real assembly, the roots of engaged threads are not sliding contacts, but rather air gaps.

In all studies, it is clear that out of ignorance or inability to disassemble an assembly without loosening it, nobody was ever interested in identifying local contact conditions when the bolt is tightened. In this study, the case of fasteners with silver coating, used in aerospace engine and breaking systems, is treated. Silver coating is a solid lubricant used in bolted joints engineering to prevent seizure and corrosion phenomenon. As a Cd coating, silver is also able to resist high temperature. The problem is then analyzed from a local point of view using experimental and numerical approaches and by adopting the concepts of the third body [17] and of the tribological triplet [18] to structure tribological experts' assessments. By remaining in a logic of non-disturbance of contact conditions, a bolted joint model is used and is disassembled by cuts (without loosening). From these assessments, a local vision of bolted structures is proposed considering the problem at the threads contacts, the most frictional and confined contacts. A particular attention is paid to the role of third body in the threads contacts and to the impact of its morphology on the self-loosening phenomenon of bolted joints. To meet this need, an approach based on DEM is used. This approach has largely shown its potential, especially when it is combined to experimental one $[19,20]$

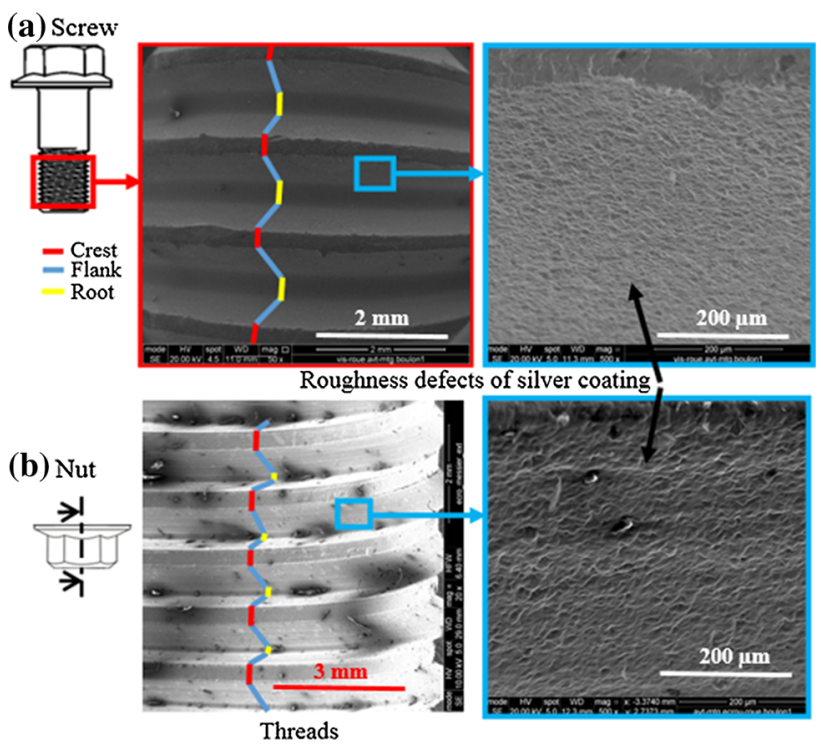

Fig. 1 Morphology of the silver coating of the screw (a) and the nut (b) before the tightening process

\section{Experimental Analysis}

As mentioned above, the experimental approach consists in looking the tightening process at the local scale. To perform the whole study, different analyses are necessary. The first one consists in looking at the nut and screw surfaces without grease in order to analyze the initial contact conditions (cf. Fig. 1). The second step is achieved by considering the grease application on threads. Scanning electron microscopy (SEM) techniques are used to perform observations.

Figure 1 shows the initial morphology of the silver coating used for both nut and screw. One can observe that it is a regular set of surface roughness defects. Such morphology will serve for tracking local contact conditions in the remainder of the study.

Then to complete these first observations, a tightening is realized without grease to analyze local contact conditions (cf. Fig. 2). The tightening consists in maintaining two plates in contact as shown in Fig. 2. The tightening is carried out until a specific torque value. After the tightening, the nut and screw are cut off (without loosening) to preserve in situ contact conditions.

Figure 2 allows to notice an important fact. Indeed, when focusing at local scale, all the thread flanks are not loaded surfaces, especially the threads roots. This observation is not in accordance with the assumptions of previous works [16], where it was considered that threads roots are submitted to sliding contacts. 


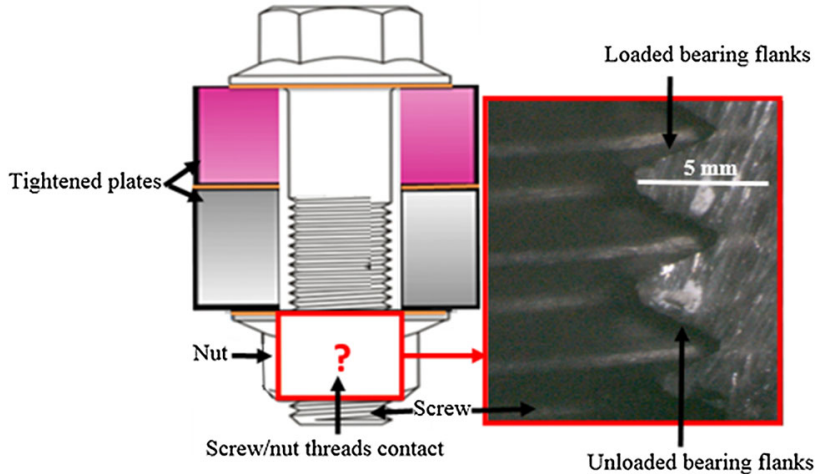

Fig. 2 Bolted joint showing contacts studied

Finally, the greased bolted joint is tightened. NYCO Grease GN GA 47 is applied on the threads of the screw before tightening phase. It serves to reduce both friction and wear of silver coating and eventual seizure. This grease is an homogenous mixture of micronized graphite $(50 \%$ by weight) and pure petrolatum vaseline (mineral wax and paraffin oil). As previously, the bolted joint is cut off without loosening. The inside of the screw threads obtained is shown in Fig. 3b.

Comparing the screw disassembled by cuts to another one freshly greased (cf. Fig. 3a), three conclusions emerge:

1. The grease has turned into clusters of particles localized on threads roots and on non-loaded bearing flanks;

2. These clusters of particles are such "dry solid";

3. There is no trace of paraffin oil, which suggests a drying up effect of the grease.

In complement of such observations, investigations on the screw surface are pursued using energy-dispersive $\mathrm{X}$-ray. Different areas of interest are chosen. With such a chemical analysis, it is possible to determine cluster composition and so to propose a possible scenario of the life of the nut/screw threads contacts in tightening process.

(a) Grease $=$ graphite ( $50 \%$ by weight $)+$ paraffin oil + mineral wax

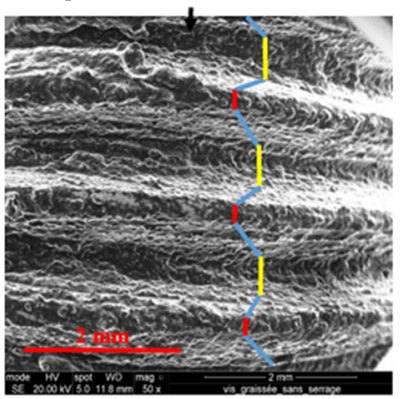

(b)

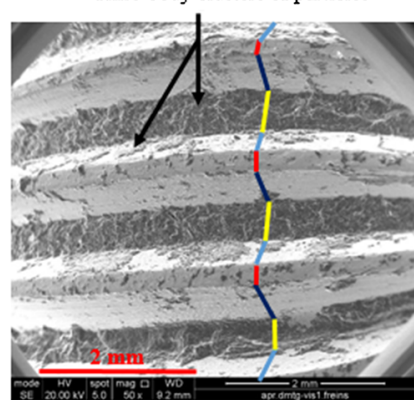

- Loaded bearing flanks

Unloaded bearing flanks

Fig. 3 Inside of screw threads a freshly greased, b after tightening process and disassembly by cuts
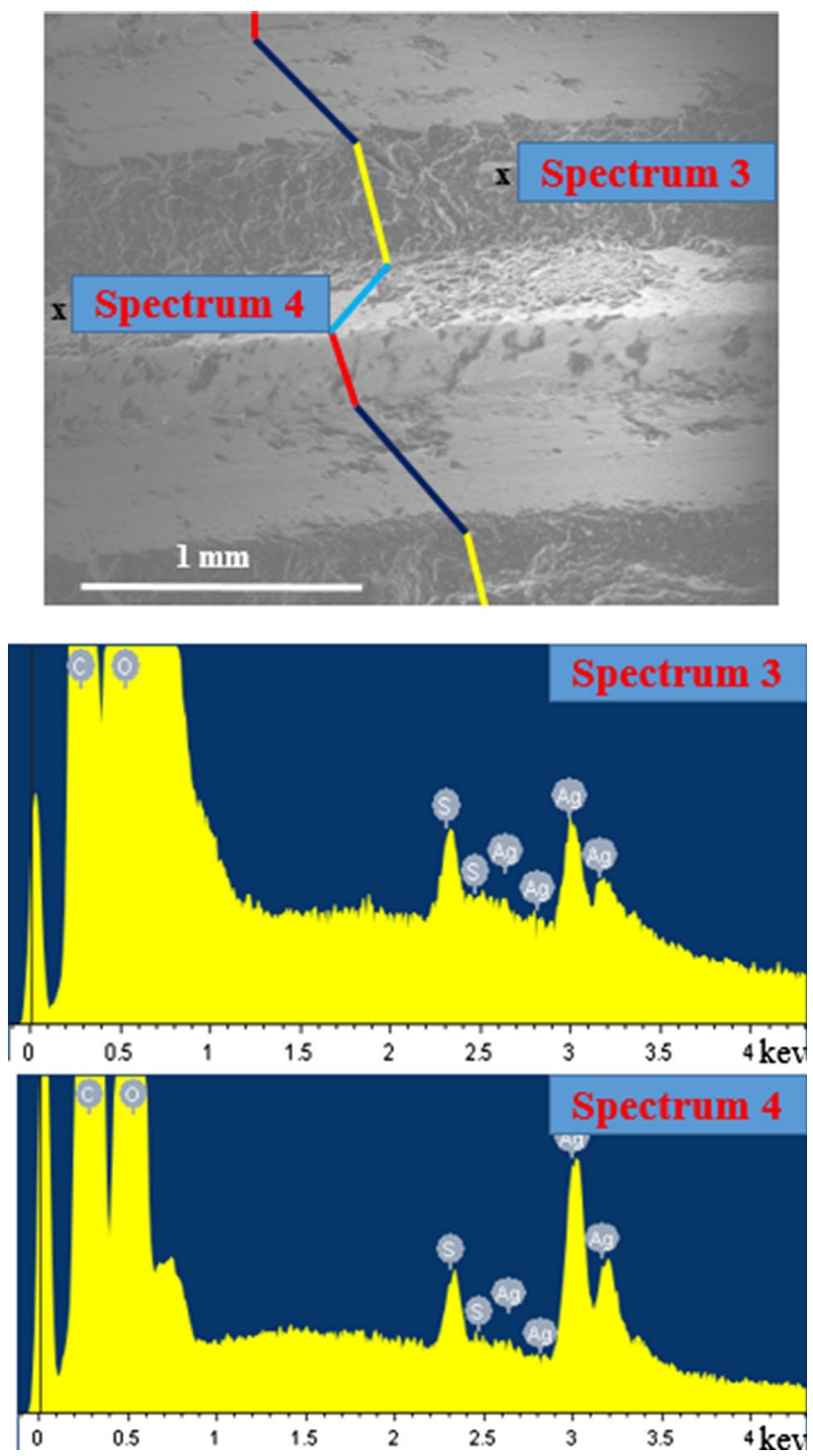

Fig. 4 Chemical analysis of particles clusters

According to spectra of area 3 and 4 (cf. Fig. 4), the clusters are composed essentially of carbon and silver. The carbon peak corresponds to the graphite contained in the grease and the silver peak corresponds to the silver coating.

To confirm such an assumption, a zoom on load-bearing flanks of the screw and a comparison with the initial flanks (before the tightening process) is done (cf. Fig. 5). One can observe that after the tightening process, the roughness of the coating disappears.

According to Fig. 5, there has been a plastic flow of silver roughnesses. Detached particles (natural third body) then formed a mixture with grease (artificial third body) initially applied to the threads of the screw. This mixture gives rise to clusters of particles by an ejecting mechanism shown in Fig. 6 and can be found in the roots and on the unloaded flanks. 
(a)

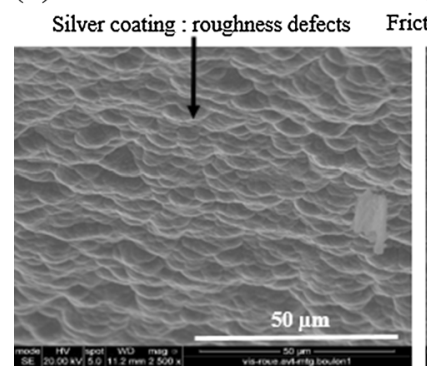

(b)

Fig. 5 Flank before tightening process (a) and after tightening and disassembly by cuts $(\mathbf{b})$

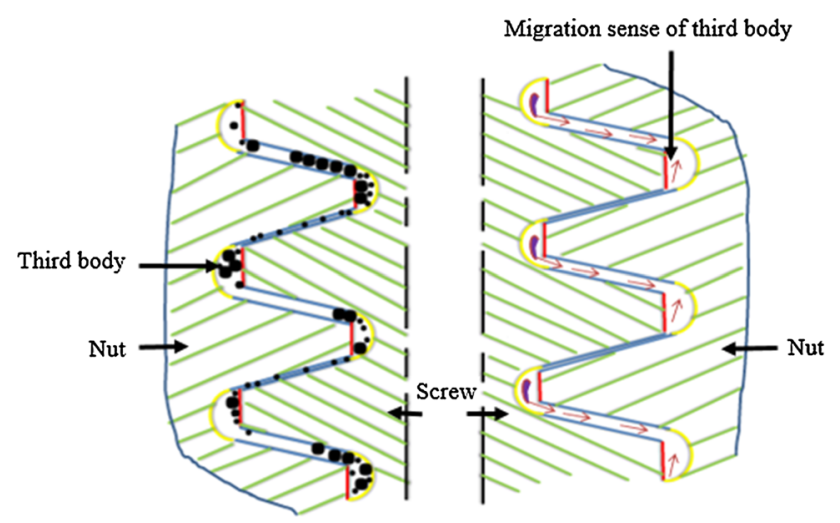

Fig. 6 Ejecting mechanism of third body from threads contacts of bolted joints during tightening process

During the formation process of the resulting third body (silver-graphite mixture), the base oil of grease would be pumped by detached particle reactivity (physicochemical phenomena), reactivity amplified by friction between the screw and the nut. Moreover, this resulting third body, with its "dry-solid" nature, is mainly spread on the loaded flanks of the nut (cf. Fig. 7), and this is reproducible over all the threads.

This experimental analysis shows that in bolt-tightening process, it is a resulting "dry-solid" third body which accommodates the velocity jump between the screw and the nut and not the grease only. According to such

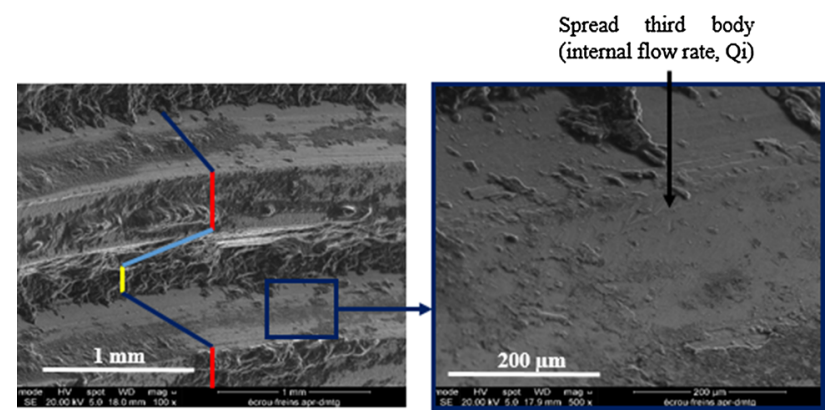

Fig. 7 Spread third body on the loaded flanks of the nut observations, one remaining question is : What is the influence of the third-body rheology on the efficiency of the clamping force and on the self-loosening phenomena?

\section{Numerical Analysis}

\subsection{Methodology}

In bolted assemblies, screw/nut threads contacts are confined and submitted to complex tribological phenomena (degradation, third-body transformation). Consequently, the modeling of these contacts is not simple because the problem practically prevents all possible simplifications (no symmetry, non-homogeneous loadings, presence of a third body, etc.) and has led modelers to propose simplified global models. As in the experimental section, a local vision is proposed hereafter to model screw/nut threads contacts (cf. Fig. 8) .

The chosen model aims at being sufficiently rich to take into account the phenomenology observed above and thus to contain necessary ingredients to give an answer to the question concerning the influence of the third body on the self-loosening phenomena. So, the essential ingredients to consider, referring to tribological triplet approach [18], are the first bodies (the screw and the nut), the third body (silver-carbon mixture) and specific boundary conditions which should reproduce as well as possible real local contact conditions.

As one wants to focus on the impact of third-body rheology, wear and degradation aspects are not taken into account in this first instance. Moreover, the plastic deformation of the Ag coating is not taken into account. Only the plastic flow of the third body is modeled. The impact of grease is considered through the cohesive interaction laws as well as the presence of carbon particles.

For the purpose of such local modeling, a discrete element method (DEM) [21] is used. Such approaches are the only way to model the heterogenous and discontinuous feature of the first body without using an a priori (a)

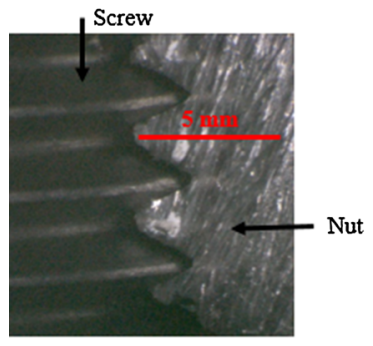

Fig. 8 a Real contacts and b proposed local model (b)

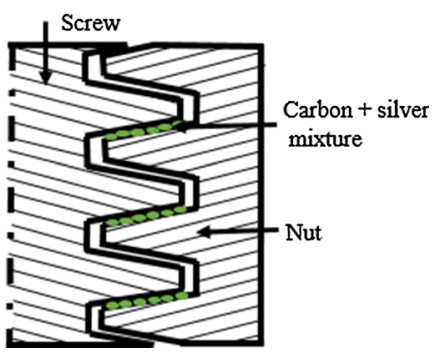


constitutive law. The chosen DEM to model the screw/nut threads contacts is the NSCD framework[22], widely used in the context of numerical tribology [23-26]. The main lines of this approach (time integration based on theta scheme, implicit contacts resolution via a nonlinear GaussSeidel algorithm) are not detailed here. Only the interaction laws for managing contacts between particles are detailed. We refer to previous works for more information [22].

To model the physicochemical interfacial phenomena that occur during the bolt-tightening process (such as the detached particles reactivity), a cohesive unilateral contact law is used, governed by the following set of inequalities:

$\left(r_{n}+\gamma\right) \geq 0 \quad g \geq 0 \quad\left(r_{n}+\gamma\right) \cdot g=0$.

The cohesive contact force, denoted $\gamma$, acts on a distance $d_{w} . r_{n}$ represents the normal contact force and $g$ the gap between particles. This contact law manages not only the interactions within the third body but also those between the third body and the first bodies.

\subsection{Model}

The proposed model is a 3D model, built from a collection of rigid spherical particles (cf. Fig. 9)

The first bodies, represented here by the skin of screw and nut, are made of 2440 silver particles each with a mean diameter equal to $20 \mu \mathrm{m}$. For the third body (i.e., the silver-carbon mixture), a representative diameter of $8 \mu \mathrm{m}$ is chosen. According to tribological experts assessments, the quantity of the third body spread on the bearing loaded flanks is not uniform. It is decreasing from the first engaged thread to the last one. Thus, in the model, the number of resulting third-body particles is set to 7200 in one of the loaded bearing threaded flanks and to 5400 in the other.

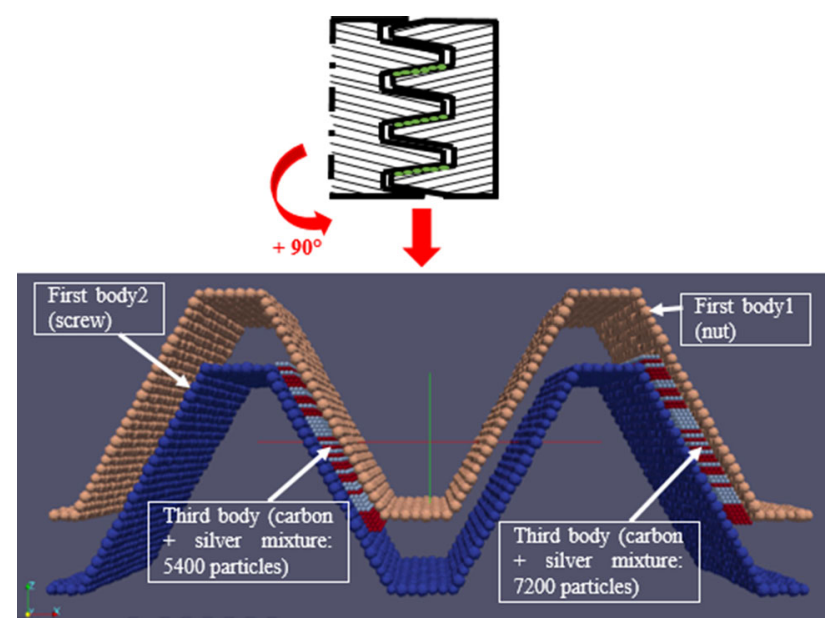

Fig. 9 Obtained model

\subsection{Numerical Simulation of Tightening Process}

The clamping mechanism of bolts is simulated by applying the preload to the upper first body in the $X$-direction and the clamping speed to the lower one in the $Y$-direction (cf. Fig. 10a). Experimentally, the preload changes gradually as the bolt is tightened (rotation of the nut or screw). This leads to the temporal evolution of various boundary conditions from the curves profile of the Fig. 10b. Indeed, the preload increases gradually and reaches a constant value at the end of tightening. In theses conditions, the clamping speed remains constant. Periodic boundary conditions are considered in the $Y$-direction ( shearing direction) in order to account for material continuity due to the helicoidal form of threads. All other degrees of freedom are blocked. The maximal value of the preload is set to $350 \mathrm{~N}$, an experimental value reduced to the size of the numerical specimen. The tightening speed is set to $2 \mathrm{~m} / \mathrm{s}$.

By simulating the tightening process, the third body, located initially on the loaded flanks, is extruded at the feet and crests of threads as materialized on Fig. 6 and shown by the arrows drawn on simulation snapshots (cf. Fig. 11a).

Then, they are amassed on threads roots and on nonloaded bearing flanks (cf. Fig. 11b). Indeed, at the end of the tightening process, one part of the third body remains in the interface (the nut was hidden on the Fig. 11c in order to clearly show the behavior of threads interface). This fits

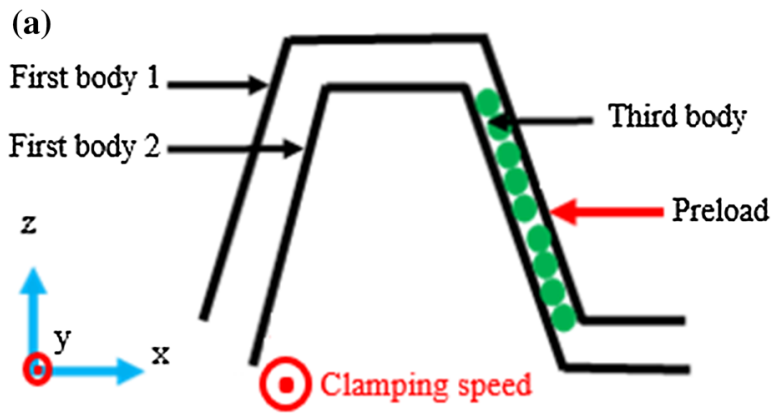

(b)

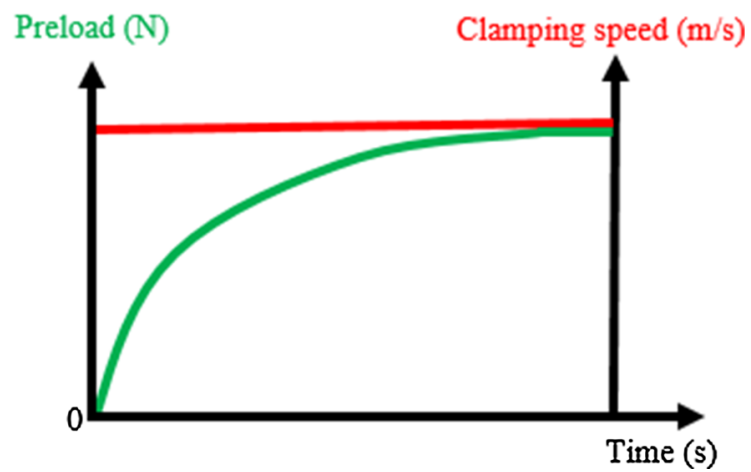

Fig. 10 a Boundary conditions, $\mathbf{b}$ timing of boundary conditions 
(a)

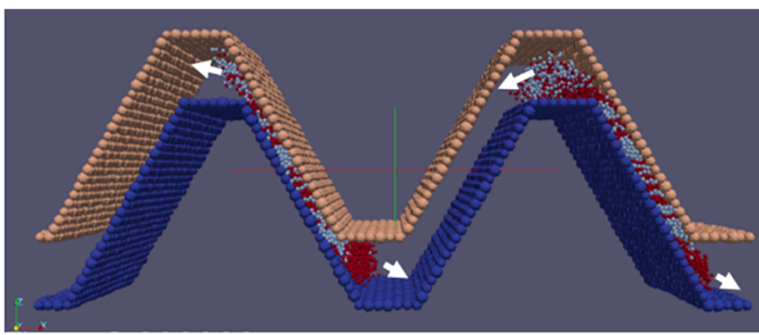

(b)

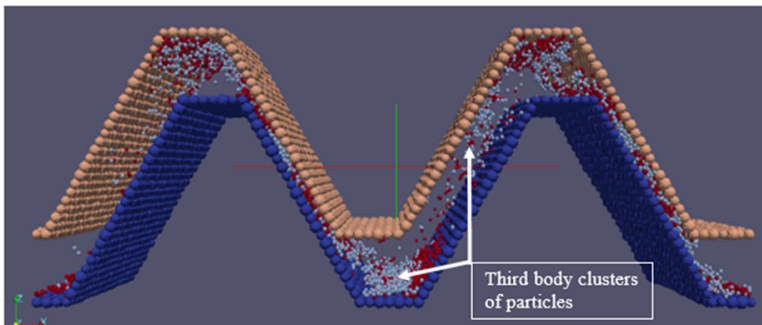

(c)

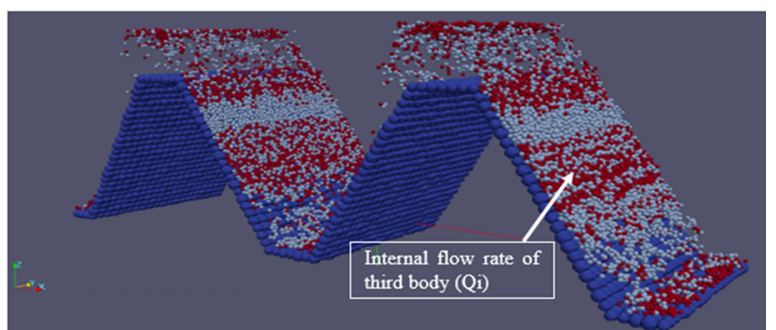

Fig. 11 Tribological phenomena of threads contacts during the tightening process: a beginning of third-body extrusion, $\mathbf{b}$ formation of clusters of particles, $\mathbf{c}$ interfacial behavior (first body 1 omitted for clarity)

perfectly with observations on real assemblies made on Fig. 3.

To confirm such static observations, evolution maps are plotted to observe the evolution in time of the third-body flow. Thus, the model is cut in 60 intervals in the $X$-direction, and during the process, the number of third-body particles in each interval is summed. Figure 12 presents such a map; the red color corresponds to the maximal value, while the blue color corresponds to the minimal value of particles number.

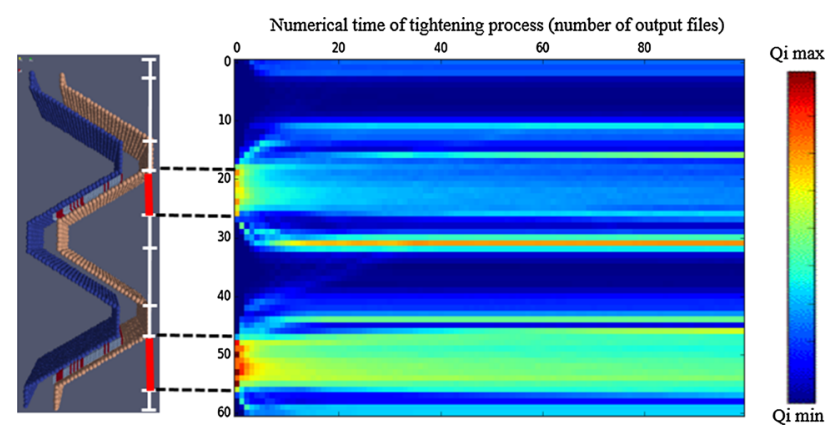

Fig. 12 Map of internal flow of third body during tightening process
The map clearly shows the evolution of third-body flow during the tightening process. In the loaded flanks, the volume of third body decreases at first before becoming constant until the end of the operation. On the contrary, it increases logically at first on the roots.

\subsection{Influence of Third-Body Cohesion}

Previous numerical observations match with experimental ones. To go further in the study of the third-body rheology, different values of the cohesion force have been chosen: $10^{-3}, 10^{-4}, 10^{-5} \mathrm{~N}$. These values are chosen in order to have $\eta \leq 1$. This status parameter, $\eta$, is defined as:

$\eta=(\gamma / s) /(F / S)$

where $\gamma / s$ is the local cohesion with $s$, the surface of one third-body particle. $F / S$ is the global cohesion with $F$, the preload, and $S$, the section of loaded bearing threads flanks of proposed model. Phenomenologically, the condition, $\eta \leq 1$, means absence of seizing up phenomena. Figure 13 presents third-body evolution maps for the three cohesion values.

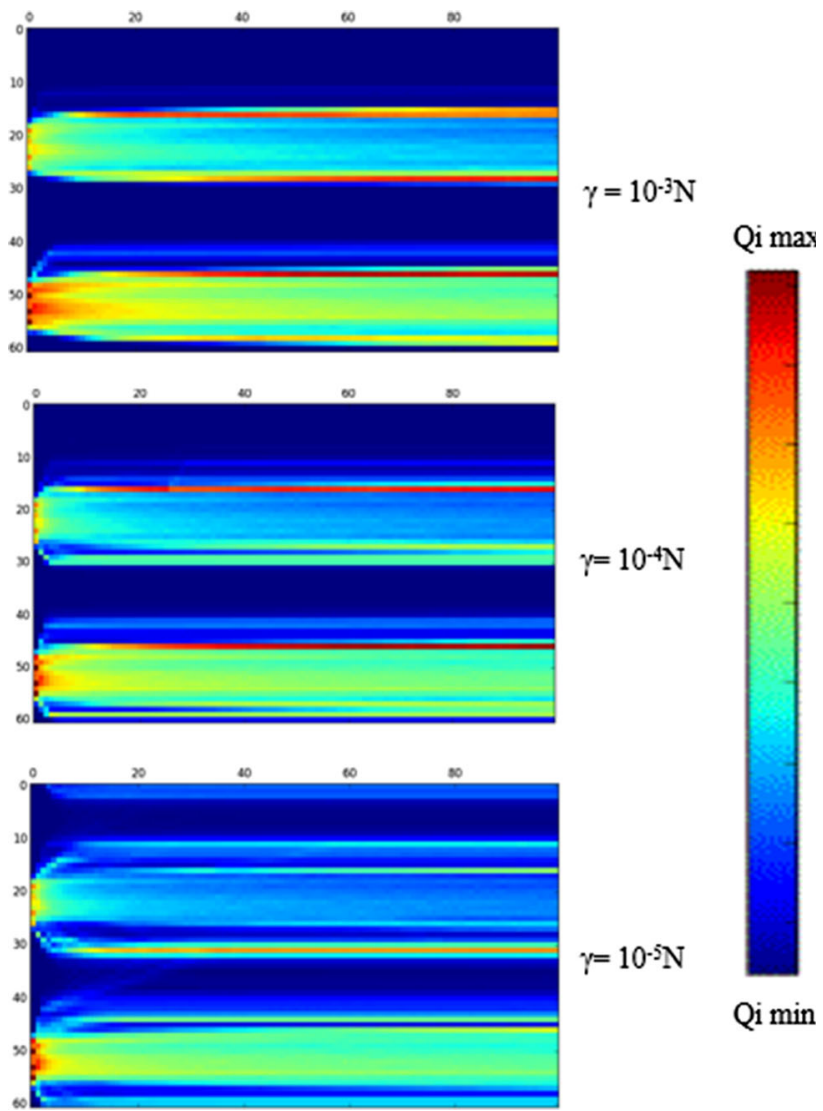

Fig. 13 Maps of internal flow according to cohesion force during the tightening phase 
It appears that when the inter-granular cohesion force is relatively small, most of the third-body particles tend to quickly eject out of the contacts. Moreover the quantity of spread third body (internal flow of third body) is larger with small cohesion value than with large cohesion value. These observations seem realistic regardless of preload and tightening speed value. One also could notice that when the cohesion is small the thickness of the spread layer of third body is more uniform. It is typically not the case with the largest value even in the loaded flanks.

To complete such observations, the evolution of the mean friction value is computed for the three values of the cohesion. The mean friction is defined as the ratio of total tangential forces by total normal forces to the bearing loaded flanks. The total tangential force results from the interaction between third body and screw particles, while the normal force is derived from the preload applied to the nut. The moving average of the signal is then plotted on the Fig. 14 to read the general trend of the system.

Hence, one can observe that, in each case, the friction value increases until reaching a value closed to 0.18 . The evolution is strongly related to the cohesion value and can be decomposed into two phases, denoted (a) and (b) on the Fig. 14. For the smaller cohesion value (i.e., $\gamma$ equal to $10^{-5} \mathrm{~N}$ ), the mean friction increases immediately during the phase (a) and reaches a stationary value with large oscillations on the phase (b). For the larger cohesion value (i.e., $\gamma$ equal to $10^{-3} \mathrm{~N}$ ), the mean friction is quasi-constant during the phase (a) and increases during the phase (b),

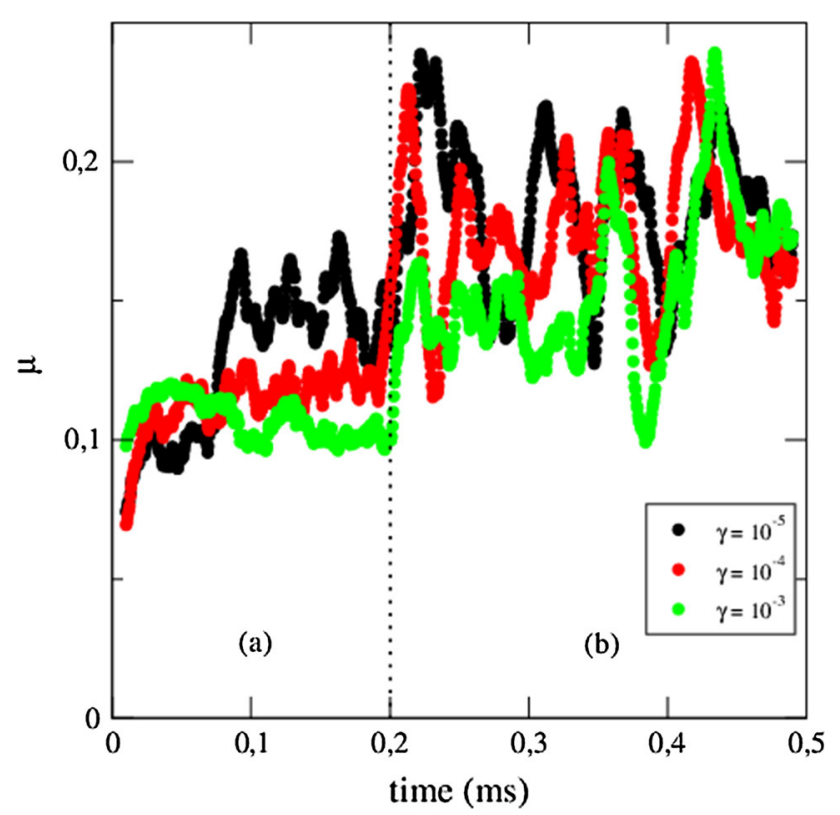

Fig. 14 Evolution of the mean friction coefficient along time during the simulation for the three cohesion values with the same oscillations than the other cohesion values. For the median cohesion value, the evolution of friction is close to the behavior of the smaller cohesion but with a smaller increase during the phase (a). Such a result can be explained by the fact that, when the cohesion is small, the third body which plays the role of lubricant is quickly ejected from the contact, leading to the increase in friction. When the cohesion is larger, the third body is ejected with more difficulty, leading to a better accommodation of the velocity and thus to slower increase in the mean friction value.

\section{Discussion}

In this present work, the coupled experimental-numerical approach has allowed to understand a certain number of phenomena in bolted joints. Experimentally, it was observed that a "dry-solid" third body is formed and remains trapped in the contacts in tightening phase. It was shown that roots in screw/nut contacts appear as unloaded and play the role of third-body trap: Once it is inside the roots, it can no more enter in the loaded flanks.

Numerical simulations confirm such tribological scenarios (extrusion, clusters of particles forming, internal flow). From there, trends emerge. When the third body is less cohesive, it is quickly extruded; this fast ejection can be explained by the fact that the forces repartition is more stable in the process. On the contrary, when the third body is cohesive (the case of silver-carbon mixture), the extrusion is slow. Indeed, the third body stays in the contact with an heterogenous forces repartition. These results suggest that under dynamical solicitations the third body will continue to be extruded and thus relax the normal load within the system.

Thus, in order to avoid self-loosening phenomenon by quick extrusion of the third body out of threads contacts with dynamic loadings, a possible way would be to increase its cohesion properties. Just as the research works of [27], numerical results also suggest that increasing these cohesion properties would reduce the threads contacts friction and hence the tightening torque value.

\section{Conclusions}

The model developed in this study does not take into account the plastic flow of coating, either the fluid lubricants (grease, etc.). This model only considers the third body formed in threads contacts during tightening solicitations. It constitutes the first step toward multiphysical modeling for understanding tribological phenomena linked to structural integrity of bolted assemblies. 
These modeling aspects, namely degradation of first bodies, taking into account of fluid lubricants, etc., constitute the challenges and prospects of these works.

Acknowledgments This work is carried out through the project FUI CARAB (Conception Avancée Robuste pour les Assemblages Boulonnés). The authors thank the assistance provided by "Fonds Unique Interministériel." Discrete element simulations have been carried out through the open-source software platform LMGC90 [28].

\section{References}

1. Chumakov, R.: Optimal control of screwing speed in assembly with thread-forming screws. Int. J. Adv. Manuf. Technol. 36(3), 395-400 (2008)

2. Martin, J.M., Donnet, C., Le Mogne, T., Epicier, T.: Superlubricity of molybdenum disulphide. Phys. Rev. B 48 , 10583-10586 (1993)

3. Wahl, K., Singer, I.: Quantification of a lubricant transfer process that enhances the sliding life of a $\mathrm{MoS}_{2}$ coating. Tribol. Lett. 1(1), 59-66 (1995)

4. Fratesi, R., Roventi, G.: Corrosion resistance of $\mathrm{Zn}-\mathrm{Ni}$ alloy coatings in industrial production. Surf. Coat. Technol. 82(1-2), 158-164 (1996)

5. Ramanauskas, R., Quintana, P., Maldonado, L., Pomès, R., PechCanul, M.: Corrosion resistance and microstructure of electrodeposited $\mathrm{Zn}$ and $\mathrm{Zn}$ alloy coatings. Surf. Coat. Technol. 92(1-2), 16-21 (1997)

6. AAIB. http://www.aaib.gov.uk/ (2006)

7. Eccles, W., Sherrington, I., Arnell, R.D.: Frictional changes during repeated tightening of zinc plated threaded fasteners. Tribol. Int. 43, 700-707 (2010)

8. Croccolo, D., De Agostinis, M., Vincenzi, N.: Failure analysis of bolted joints: effect of friction coefficients in torque/preloading relationship. Eng. Fail. Anal. 18(1), 364-373 (2011)

9. Junker, G.: New criteria for self-loosening of fasteners under vibration. SAE Paper 690055 (1969)

10. Kellermann, R., Klein, H.C.: Untersuchungen über den Einflu $\beta$ der Reibung auf Vorspannung und Anzugsmoment von Schraubenverbindungen. Springer, Berlin (1955)

11. Motosh, N.: Development of design charts for bolts preloaded up to the plastic range. ASME J. Eng. Ind. 98, 849-851 (1976)

12. Shigley, J.E., Mischke, C.R.: Screws, Fasteners, and the Design of Nonpermanent Joints. In: Budynas, R.G., Nisbett, J.K. (eds.)
Mechanical engineering design, pp. 410-506. McGraw-Hill, New York (1989)

13. Juvinall, R.C., Marshek, K.M.: Fundamentals of Machine Component Design. Wiley, New York (2000)

14. Nassar, A., Yang, X.: Novel formulation of the tightening and breakaway torque components in threaded fasteners. ASME J. Press. Vessel Technol. 129, 653-663 (2007)

15. Mackerle, J.: Finite element analysis of fastening and joining: a bibliography. Int. J. Press. Vessels Pip. 80, 253-271 (2003)

16. Jiang, Y., Zhang, M., Lee, C.H.: A study of early stage selfloosening of bolted joint. ASME J. Mech. Des. 125, 518-526 (2003)

17. Godet, M.: The third-body approach: a mechanical view of wear. Wear 100, 437-452 (1984)

18. Berthier, Y., Vincent, L., Godet, M.: Velocity accommodation in fretting. Wear 125, 25-38 (1988)

19. Descartes, S., Renouf, M., Fillot, N., Gautier, B., Descamps, A., Berthier, Y., Demanche, P.: A new mechanical electrical approach to the wheel-rail contact. Wear 265(9-10), 1408-1416 (2008)

20. Charlery, R., Renouf, M., Saulot, A., Daly, N., Berthier, Y.: Experimental and numerical modelling of the ignition of solid propellant. Tribol. Int. 82, 330-342 (2015)

21. Radjai, F., Dubois, F. (eds.): Discrete-Element Modeling of Granular Materials. Wiley-ISTE, London (2011)

22. Jean, M.: The non-smooth contact dynamics method. Comput. Methods Appl. Mech. Eng. 177, 235-257 (1999)

23. Renouf, M., Fillot, N.: Coupling electrical and mechanical effects in discrete element simulations. Int. J. Numer. Meth. Eng. 74(2), 238-254 (2008)

24. Champagne, M., Renouf, M., Berthier, Y.: Modeling wear for heterogeneous bi-phasic materials using discrete elements approach. ASME J. Tribol. 136(2), 021603 (2014)

25. Riviere, J., Renouf, M., Berthier, Y.: Thermo-mechanical investigations of a tribological interface. Tribol. Lett. 58(3), 1023 (2015)

26. Mollon, G.: A numerical framework for discrete modelling of friction and wear using Voronoi polyhedrons. Tribol. Int. 90, 343-355 (2015)

27. Iordanoff, I., Fillot, N., Berthier, Y.: Numerical study of a thin layer of cohesive particles under plane shearing. Powder Technol. 159, 46-54 (2005)

28. Dubois, F., Jean, M.: LMGC90: une plateforme de développement dédiée à la modélisation des problèmes d'interaction. In: Actes du sixième colloque national en calcul des structures, Giens, France (2003) 